\title{
Implementation of a toxic cuprizone model of demyelination in vitro
}

\author{
Rodnichenko A. E. \\ State Institute of Genetic and Regenerative Medicine of the National Academy of Medical Sciences of Ukraine, Kyiv, Ukraine \\ e-mail: arodnichenko@ukr.net
}

\section{ABSTRACT}

The relevance of the use of adequate experimental models to study the mechanisms of the development of multiple sclerosis and the effects of drugs on the remyelination of nerve fibers due to the prevalence of this disease, which affects mostly young people.

THE PURPOSE of the study was to adjust a toxic cuprizone model in vitro, with confirming the processes of demyelination of nerve fibres.

MATERIALS AND METHODS. To study the features of the processes of myelination and demyelination of neurons axons, the culture of dissociated cerebellar cells of 7-day old newborn FVB/N mice was used. To detect myelin sheaths, we used a histochemical staining with Sudan Black $B$ dye. To identify oligodendrocytes, their culture was stained by immunocytochemistry for Olig2 marker.

RESULTS. Having developed a model of toxic demyelination, we determined the density of the cerebellar cell culture, selected conditions for the cultivation of dissociated cerebellar cells, and the survival of the dissociated culture of the cerebellum until the 26-28 th day of cultivation was achieved. Using histochemical staining, the presence of myelination and demyelination processes in vitro was confirmed. Using immunocytochemical staining, it has been shown that the neurotoxin cuprizone results in a decrease of Olig2-positive oligodendrocytes, when it is applied on the $18^{\text {th }}$ day of cultivation of the dissociated cerebellar cells culture.

CONCLUSION. The conditions for the cultivation of murine dissociated cerebellar cells were selected, the presence of myelination and demyelination processes in vitro was confirmed; a decrease in the number of Olig2-positive cells when the demyelination agent was added to the culture on the $18^{\text {th }}$ day was shown.

KEY WORDS: cuprizone; cerebellum; oligodendrocytes; demyelination

Demyelinating diseases are the most common diseases of the central and peripheral nervous system, in which the main pathomorphological manifestation is the destruction of the myelin sheath of nerve fibres and, as a result, the violation of the nerve impulses and motor activity [1-3].

Among the most common demyelinating diseases of the central nervous system, which affect mainly young people, is multiple sclerosis [4]. According to current concepts, multiple sclerosis is a multifactorial disease, one of the internal causes of which is the presence of a certain genetic (immunogenetic) predisposition, autoimmune and hormonal (especially steroid) disorders [2]. To study the mechanisms underlying the development of multiple sclerosis and obtain information on the adverse effects of toxic agents or carry out screening of certain medicinal compounds and develop effective therapeutic strategies, relatively simple models are used both in vivo and in vitro.

There are inflammatory, toxic, viral, and other experimental models of multiple sclerosis. Among the inflammatory models, as the standard for the development of therapeutic strategies and the testing of drugs for autoimmune diseases, the most commonly used model is experimental allergic encephalomyelitis. The viral model, Theiler's murine encephalomyelitis, involves the injection of single-stranded RNA virus in the central nervous system, resulting in immune-mediated death of oligodendro- cytes [5]. Among toxic models, a model of combined injections of toxins into the spinal cord or white matter of the brain is known. With this model, demyelination is performed in certain parts of central nervous system by inducing the death of oligodendrocytes using ethidium bromide and solubilization of myelin membrane using lysolecithin at the injection site [5].

The cuprizone model of demyelination attracts special attention among toxic models. Cuprizone [bis(cyclohexanone)oxaldihydrazone] is a copper chelator. After peroral intake, apoptosis of mature oligodendrocytes is observed and, as a result, there is demyelination of neurons axons, damage to mitochondria of oligodendrocytes (by inhibiting cytochrome oxidase, monoamine oxidase), activation of cerebral microglia (due to increased production of tumor necrosis factor-alpha and interferon-gamma). The cuprizone application allows to simulate acute and chronic forms of demyelination, and to study the agents that influence de- and remyelination [6-10].

At present, cultivation of cells and tissues is one of the most advanced research methods used in biochemistry, cellular and molecular biology. The main advantages of the nervous tissue culture and the modelling of its damage in vitro are the study of the mechanisms of neurohistogenesis, the interaction of nerve and glial cells, the direct study of the cell culture using microscopic and electrophysiological research methods 
[2]. There is no doubt that in the in vitro system it is impossible to reproduce all processes that are observed on models in vivo. However, in vitro models are useful ones that allow evaluating cell functions and processes that occur at different stages of their development, and the direct effect of both demyelinating and remyelinating agents. In addition, on the models in vitro, it is possible to study the mechanisms associated with changes in myelination and some aspects of the interaction of cells and the effect of this interaction on the development of oligodendrocytes and, accordingly, myelination. These models also have a decisive advantage, first, in reducing the number of animals used in experiments in vivo.

On models in vivo (different mouse strains, Wistar rats), it was shown that after cuprizone application for 4 weeks demyelination of such parts of the brain as the corpus callosum, cerebellum, hippocampus, cortex, and white matter of the spinal cord occurs [8, 11-15]. The cerebellum has the largest number of fibrous structures in the mammalian brain, which makes it an efficient and useful object for the study of myelination [16].

Therefore, the purpose of this work was to confirm the availability of myelination processes of nerve fibres and investigate the direct effect of the demyelinating agent cuprizone on the model of murine dissociated cerebellar cells in vitro.

\section{MATERIALS AND METHODS}

All works with experimental animals were carried out in compliance with the legislation and principles of bioethics: the Law of Ukraine «On the Protection of Animals from Cruel Treatment» (dated February 21, 2006), «European Convention for the Protection of Vertebrate Animals Used for Experimental and Other Scientific Purposes» (European Convention, Strasbourg, 1986). To study the features of myelination and demyelination of axons, the culture of dissociated cerebellar cells of the 7-day-old FVB/N mice $(n=27)$ was used. The mice were euthanized with an overdose of diethyl ether.

Preparation of the cerebellum was carried out in sterile conditions, on ice. The cerebellum was placed in the medium containing $90 \%$ of the DMEM growth medium, $10 \%$ horses serum, inactivated at $56^{\circ} \mathrm{C}$ for 30 minutes, glucose $(6 \mathrm{~g} / \mathrm{L}), 100 \mathrm{U} / \mathrm{mL}$ penicillin, and $100 \mu \mathrm{g} / \mathrm{mL}$ streptomycin (all - Sigma, USA). Dissociated cells suspension were obtained by minced the cerebellum with scissors in $0.25 \%$ trypsin solution (Sigma, USA) after pre-washing samples in phosphate buffer saline (PBS) with antibiotics PenStrep (Sigma, USA). The resulting suspension was incubated for 5 minutes at $+37^{\circ} \mathrm{C}$ on shaker, followed by mechanical dissociation using Pasteur pipettes of different diameters. The dissociated cerebellar cells were counted and transferred to $35 \mathrm{~mm}$ Petri dishes precoated with poly-L-lysine, with seeding density of $3 \cdot 10^{5} \mathrm{cells} / \mathrm{cm}^{2}$ and $2 \cdot 10^{6} \mathrm{cell} / \mathrm{s} / \mathrm{cm}^{2}$. Cell cultivation was performed in complete growth medium that contained $90 \%$ DMEM, $10 \%$ horse serum, $6 \mathrm{~g} / \mathrm{L}$ glucose, $10 \mu \mathrm{g} / \mathrm{mL}$ bovine insulin, $100 \mathrm{U} / \mathrm{mL}$ penicillin, and $100 \mu \mathrm{g} / \mathrm{mL}$ streptomycin (all - Sigma, USA) under standard conditions in a $\mathrm{CO}_{2}$-incubator at $+37^{\circ} \mathrm{C}$ and a humidified atmosphere with $5 \% \mathrm{CO}_{2}$.

At the second stage of the study, after a week of cultivation in a cerebellar cell culture, cuprizone (Sigma, USA) was added at a dose of $25 \mu \mathrm{M}$ or $500 \mu \mathrm{M}$ for two hours and then the medium was replaced. At the third stage, cuprizone at a dose $25 \mu \mathrm{M}$ with a longer time of incubation for $48 \mathrm{~h}$ was used. To clarify the effect of neurotoxin at different stages of the development of oligodendrocytes, cuprizone was added on the $7^{\text {th }}$ or $18^{\text {th }}$ day of cultivation. The cultivation was carried out within the $26-28^{\text {th }}$ days with the change of the growth medium twice a week. The cell culture were investigated using an inverted microscope IX71 (Olympus, Japan) at phase contrast mode.

To detect myelin sheaths, we used a histochemical method of their staining using a Sudan Black B dye. The cerebellar cells at the 26-28-day of culture were washed with PBS and fixed with $1 \%$ paraformaldehyde (PFA) solution in $0.1 \mathrm{M}$ PBS for 1 hour. After that, dehydration was carried out in serial aqueous ethanol dilutions ( $25 \%, 50 \%$ and $70 \%$ ) for
5 minutes in each. Staining was performed with $0.5 \%$ Sudan Black B (Chemapol Nordic, Czech Republic) in $70 \%$ ethanol for 1 hour. The cells were destained in $70 \%$ ethanol for 30 seconds and rehydrated in $50 \%$ and $25 \%$ ethanol for 5 minutes. The stained preparations were studied using the inverted microscope IX71 (Olympus, Japan) at phase contrast mode.

To identify oligodendrocytes, we performed immunocytochemical staining of the culture for oligodendroglial lineage marker Olig2. After 28 days of cultivation, the cultures were fixed with $4 \%$ PFA solution for 30 minutes. After washing, the cell culture was blocked in $0.1 \mathrm{M}$ PBS (pH 7.4) with $0.5 \%$ bovine serum albumin and $0.3 \%$ Triton X-100 (Sigma, USA). During 48 hours at $+4{ }^{\circ} \mathrm{C}$, cell culture was incubated with primary monoclonal rabbit antibody to Olig2 (Chemicon, USA) at 1:200 dilution. The Alexa Fluor 488 anti-rabbit secondary antibody (Invitrogen, USA) at 1:1000 dilution was used. Cell nuclei were stained with Hoechst 33342 fluorescent dye (Invitrogen, USA) at 1:500 dilution. Studies were performed using Axio Observer A1 inverted fluorescence microscope equipped with AxioCam ERc 5s digital camera with ZEN 2012 and AxioVision 4.8 software, using the fluorescence filter set $21 \mathrm{HE}$ (for Hoechst 33342) and $56 \mathrm{NE}$ (for Alexa Fluor 488) (Zeiss, Germany). Cells counting was performed in five random fields of view of the culture in each experimental group.

Statistical analysis was performed using the Student's t-test in Statistica 7.0 software (StatSoft Inc., USA). The differences were considered statistically significant at $p<0.05$.

\section{RESULTS AND DISCUSSION}

Stage I - confirmation of myelination process. The advantage of neurons and glial cells culture is the ability to study the dynamics of their morphological and functional changes [2, 17]. At the first stage of the work to study the process of myelination in vitro for further study of the direct effect of the demyelinating agent on it, we selected the conditions of cultivation of dissociated cerebellar cells of newborn mice. In particular, the cell suspension density was determined and the presence of myelination processes was confirmed.

Since dissociation of the cerebellum tissue is accompanied by changes in the shape of the cells, damage and death of both neurons and glial cells (see Fig. 1), the most cells were round-shaped without processes after dissociation. The attachment of cells to the substrate occurred in the first four hours of cultivation.

After 20 hours of cultivation and during the following day, we observed the beginning of the formation of processes (Fig. 2).

As Fig. 3 shows, during the $4-6^{\text {th }}$ days of cultivation, the cells processes formed a network. Such a pattern persisted throughout the entire period of cultivation of dissociated cerebellar cells (Fig. 4).

For myelination of axons, the presence of mature differentiated oligodendrocytes is required. Oligodendrocytes are perineuronal cells of the central nervous system, which myelinise the axons and enhance the nerve impulse conduction [18]. They play an important role in maintaining the viability and function of the axons. It should be noted that one oligodendrocyte may myelinise up to 50 different axons. Myelin-productive oligodendrocytes produce up to $5-50 \cdot 10^{3} \mu \mathrm{m}$ of membrane per day. The support of the myelin membrane consists of the constant formation of myelin, correlated with high expression of corresponding genes [19]. In addition, only axons with a diameter greater than $0.2 \mu \mathrm{m}$ have myelin sheaths [20].

At this stage, the identification of oligodendrocytes was performed morphologically using a phase-contrast microscope. Oligodendrocytes have a spherical or polygonal soma and numerous thin branching processes [17]. In 1968 Bat et al., according to the morphological features and size of oligodendrocytes or the thickness of the myelin sheath, identified four types of these myelin-synthesizing cells [21]. Type I - small size cells that form thin myelin sheath and cover from 15 to 30 axons of a small diameter. Type II and III - intermediate types and type IV large cells that form long and thick myelin sheath and cover from 1 to 3 axons of a large diameter. Mory and Leblond (1970) classified three types 
of oligodendrocytes using staining of microglia with silver carbonate: light, medium and dark [22]. Dark oligodendrocytes have the compact cytoplasm under electron microscopes. The authors suggest that light oligodendrocytes actively divide and darken when they become mature [23]

At the initial identification of oligodendrocytes in the culture of cerebellar cells on the $6^{\text {th }}$ day of cultivation (regardless of the cell culture density), we have identified cells that morphologically may be immature oligodendrocytes (Fig. 5).

Since the main function of oligodendrocytes is the formation of the myelin sheath around the axons, we visualized the myelin sheaths, dyeing these structures with Sudan Black B. As can be seen from Fig. 6, we found myelinated fibres in a 26-day culture of dissociated cerebellar cells. Since myelination processes are not possible without mature oligodendrocytes, we can argue that the detection of myelin sheath of axons indicates the presence of mature oligodendrocytes in a 26-day culture of murine dissociated cerebellar cells. Despite the fact that we observed myelination processes of neurons axons at different densities of cell suspension, at the same time, while using $2 \cdot 10^{6} \mathrm{cells} / \mathrm{cm}^{2}$, we observed more myelinated axons of neurons in the field of view.

Thus, at this stage of the research it can be assumed that the studied culture of dissociated cerebellar cells undergoes a myelination, which is impossible without mature oligodendrocytes.
At the second stage of our research to study the demyelination in vitro, we used a demyelinating agent cuprizone. At the first stage of our research, already on the $6^{\text {th }}$ day of cultivation at the initial identification of cells in the dissociated culture of the cerebellum, we have identified cells that morphologically are possible oligodendrocytes. To study the direct effect of the demyelinating agent, cuprizone at doses of $25 \mu \mathrm{M}$ and $500 \mu \mathrm{M}$ was added for two hours on the $7^{\text {th }}$ day of cultivation. As Fig. 7 shows, with the application of cuprizone at a dose $25 \mu \mathrm{M}$, partial demyelination of nerve fibres was observed: both myelinated and demyelinated nerve fibres were identified. It is known from the literature data that the use of the cuprizone model results in the death of oligodendrocytes followed by demyelination. The treatment of cells with different concentrations of cuprizone does not affect the proliferation and survival of oligodendrocytes precursors, while cuprizone has a significant effect on mature oligodendrocytes with morphological changes in mitochondria (increase in size or swelling) [24] and reduction of mitochondrial potential [25]. Toxicity has already been observed at a dose of $25 \mu \mathrm{M}$. That is, the mature oligodendrocytes are the main target for the cuprizone, whereas other glial cells, including the precursors of oligodendrocytes, do not change or change slightly. With an increase in the dose of cuprizone to $500 \mu \mathrm{M}$, there was a complete demyelination of nerve fibres of 26-day culture of murine dissociated cerebellar cells (Fig. 8).
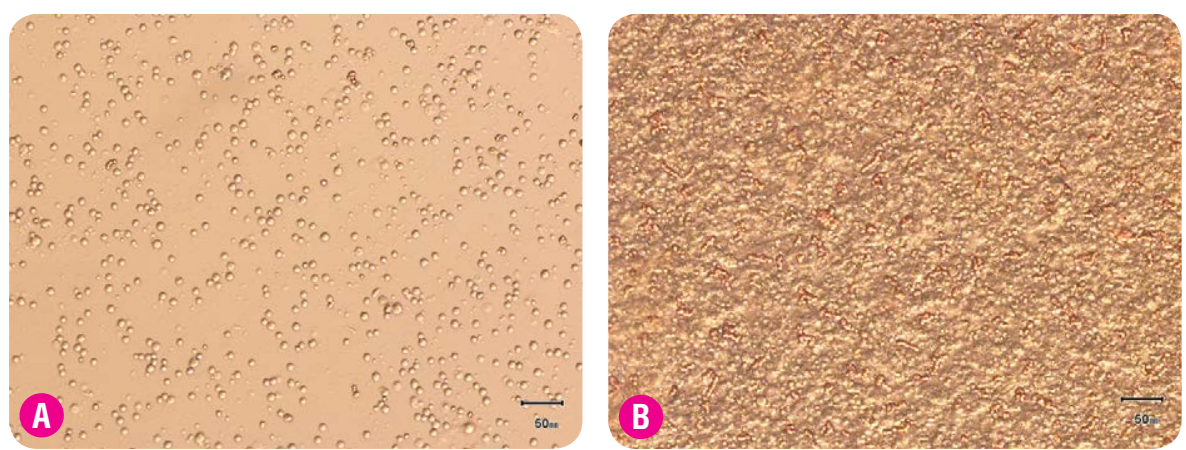

Fig. 1. Microphotographs of dissociated culture of murine cerebellar cells at 4 hours of cultivation; phase contrast. The seeding density of the cell suspension is $3 \cdot 10^{5}$ cells $/ \mathrm{cm}^{2}(A)$ or $2 \cdot 10^{6} \mathrm{cells} / \mathrm{cm}^{2}(\mathrm{~B})$.
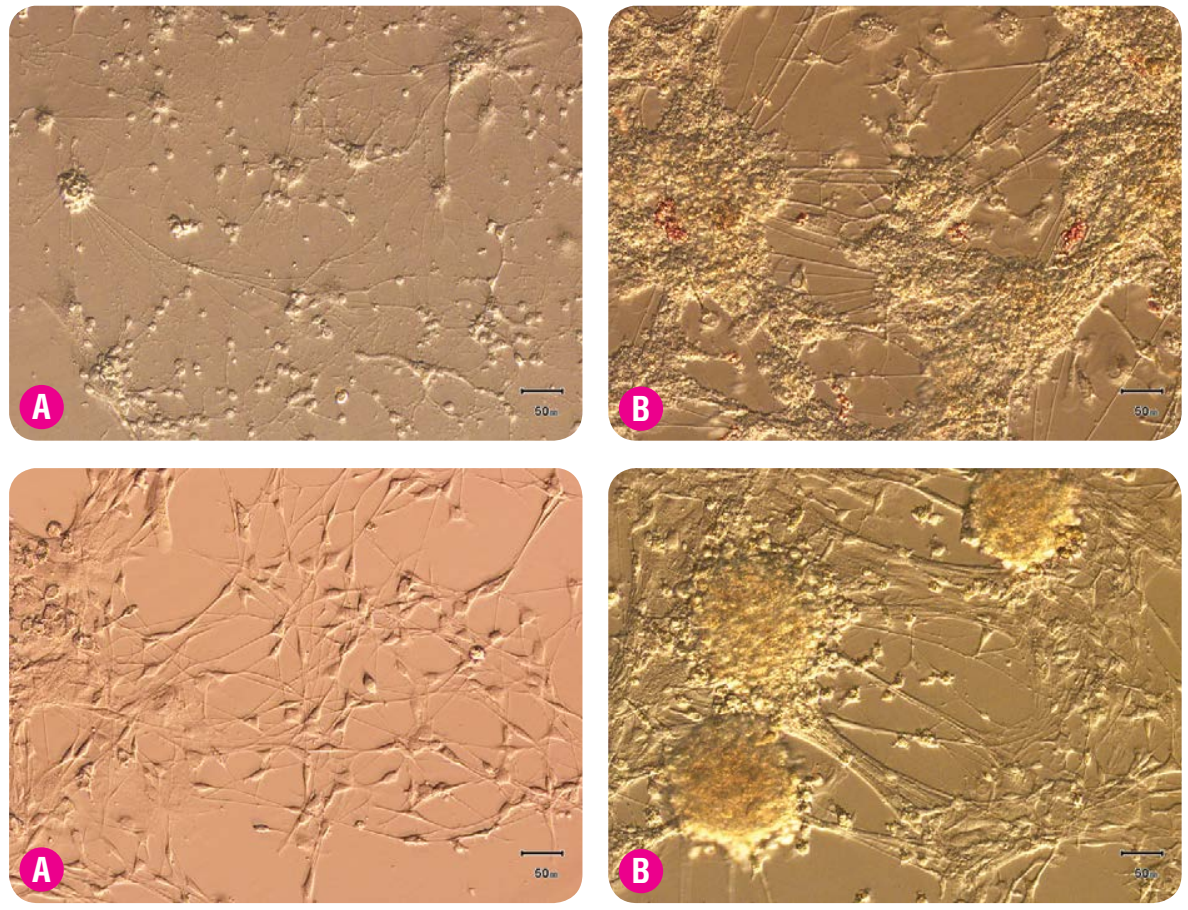

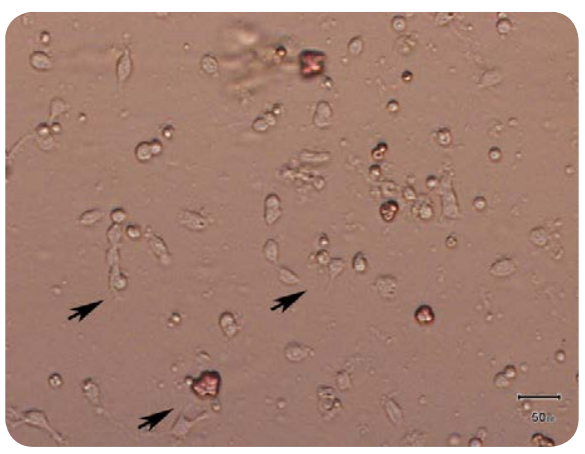

Fig. 2. Microphotograph of dissociated culture of murine cerebellar cells, 20 hours of cultivation; phase contrast. Arrows show the formation of processes.

\ Fig. 3. Microphotographs of dissociated culture of murine cerebellar cells, the $6^{\text {th }}$ day of cultivation; phase contrast. The seeding density of the cell suspension is $3 \cdot 10^{5}$ cells $/ \mathrm{cm}^{2}(\mathrm{~A})$ or $2 \cdot 10^{6}$ cells $/ \mathrm{cm}^{2}(\mathrm{~B})$.

Fig. 4. Microphotographs of dissociated culture of murine cerebellar cells, the $15^{\text {th }}$ day of cultivation; phase contrast. The seeding density of the cell suspension is $3 \cdot 10^{5}$ cells $/ \mathrm{cm}^{2}(\mathrm{~A})$ or $2 \cdot 10^{6} \mathrm{cells} / \mathrm{cm}^{2}(\mathrm{~B})$. 
Thus, the results of the cytochemical study confirmed the presence of demyelination under the influence of the cuprizone in vitro. The dosedependent demyelinating effect of the cuprizone on the axons in the murine dissociated culture of the cerebellar cells was established upon the application of cuprizone on the $7^{\text {th }}$ day of cultivation.

At the third stage of the study of the influence of cuprizone on oligodendrocytes that are at different stages of its development, we compared the demyelinating effect of the cuprizone on dissociated cerebellar cell culture at different times, namely on the $7^{\text {th }}$ and $18^{\text {th }}$ days of cultivation. At this stage of the study, we conducted an immunocytochemical staining with a lineage marker of oligodendrocytes Olig2.

Before the final maturation, oligodendrocytes pass four stages of the development. The number of phenotypic stages has been determined based on the appearance of various markers, as well as the mitotic and migration status of these cells. Oligodendrocyte precursor cells (OPCs) develop in the ventral part of the neuroepithelium of the neural tube at the early stages of embryogenesis, more precisely, from the motor neuron progenitor domain (pMN), and in the spinal cord and in the telencephalon at the later stages of the embryogenesis and early postnatal life. These proliferative cells migrate to a developing white matter, exit the cell cycle, differentiate into mature oligodendrocytes, and begin to express the myelin-associated proteins [5].
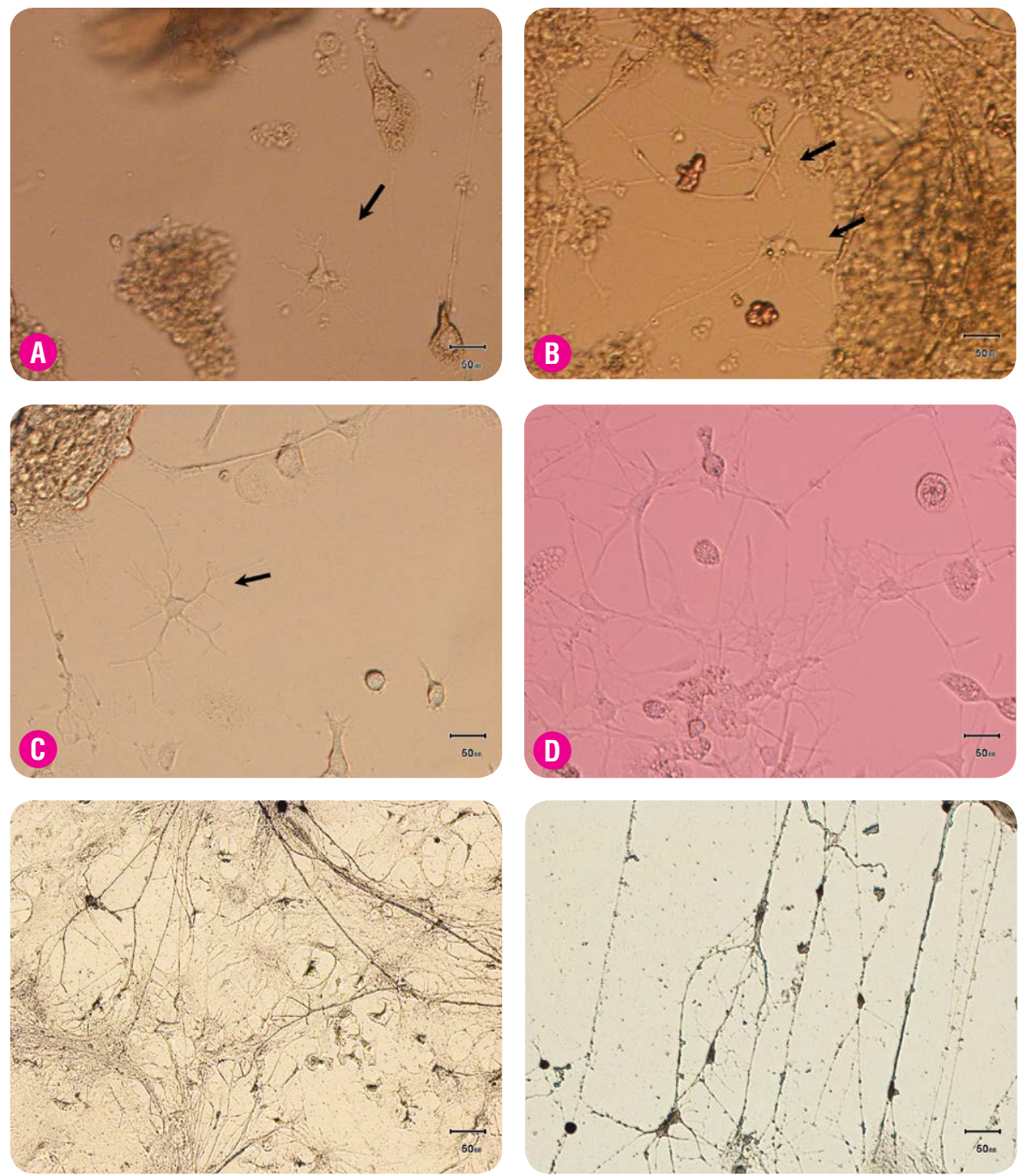

A

Fig. 6. Microphotograph of dissociated culture of murine cerebellar cells, the $26^{\text {th }}$ day of cultivation; phase contrast. Staining of myelin shells with Sudan Black B.

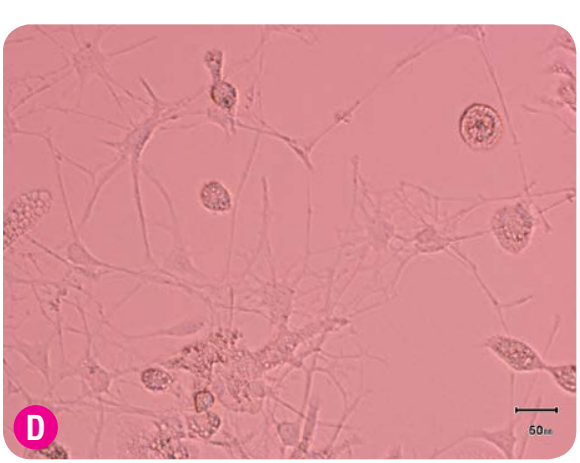

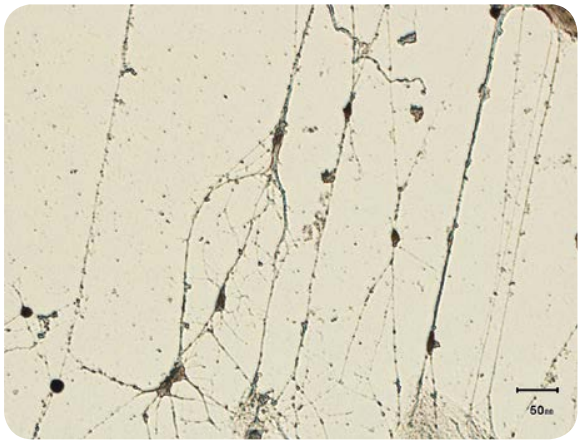

Fig. 7. Microphotograph of dissociated culture of murine cerebellar cells under cuprizone application at a dose of $25 \mu \mathrm{M}$, the $26^{\text {th }}$ day of cultivation; phase contrast. Staining of myelin shells with Sudan Black B.
Regarding the differentiation stages, oligodendrocyte precursors have bipolar morphology and express the specific markers ganglioside GD3 and NG2 (neuron-glial antigen 2) proteoglycan. These cells are actively proliferating and possess migration properties. The proliferative potential of these cells in vitro increases under application of FGF (fibroblast growth factor) and PDGF (platelet-derived growth factor).

The next stage of maturation of oligodendrocytes is the differentiation into pre-oligodendrocytes, which retain proliferation ability and express the oligodendrocyte marker 04 . At this stage, these cells are inactive and lose a proliferative response to PDGF. Then pre-oligodendrocyte differentiates into immature oligodendrocyte. This stage is characterized by the expression of galactocerebrosides (GalC/01) and the loss of expression of GD3 antigens on the cell surface. Mature oligodendrocytes express cellspecific galactocerebrosides GalC, sulfatides SUL, myelin-specific proteins CNP (2,3-cyclic nucleotide-3-phosphohydrolase), a marker of oligodendrocytes maturation PLP (proteolipid protein), a marker of oligodendrocytes differentiation MBP (myelin basic protein), MOG (myelin oligodendrocyte glycoprotein), one of the early myelin-specific proteins MAG (myelin-associated glycoprotein) [23]. MBP-positive oligodendrocytes are classified into three categories: non-membranous, membranous, and with myelin sheets. The formation of myelin sheets is described only at the later stages of the differentiation of oligodendrocytes [26, 27, 28].

Fig. 5. Microphotographs of dissociated culture of murine cerebellar cells, the $6^{\text {th }}$ day of cultivation; phase contrast. The seeding density of the cell suspension is $3 \cdot 10^{5} \mathrm{cells} / \mathrm{cm}^{2}(\mathrm{~A}, \mathrm{~B})$ or $2 \cdot 10^{6} \mathrm{cells} / \mathrm{cm}^{2}$ $(C, D)$. The arrows indicate immature oligodendrocyte-like cells.

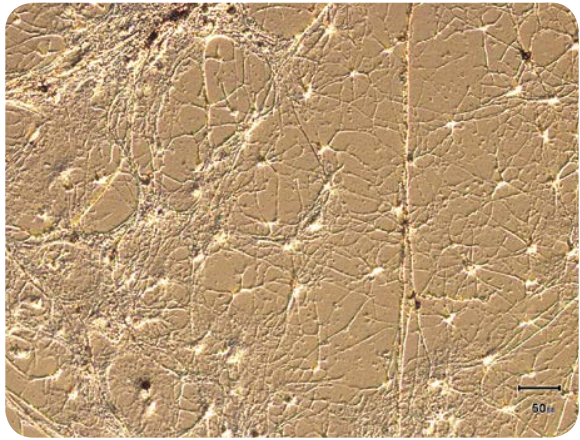

Fig. 8. Microphotograph of dissociated culture of murine cerebellar cells under cuprizone at a dose of $500 \mu \mathrm{M}$, the $26^{\text {th }}$ day of cultivation; phase contrast. Staining of myelin shells with Sudan Black B. 
Both myelination and remyelination occur through the activation of myelin-protein genes, which are induced by various transcription factors. Sequential activation of myelin protein correlates with the activation and inhibition of several transcription factors such as Olig1, Olig2 and Nkx2.2 [29]. Olig2 is required to support the expression of the MYRF (myelin regulatory factor), which regulates the myelin-bound proteins MBP, PLP1 (proteolipid protein 1), MAG, CNP and MOG, which are critical for the formation of myelin [18]. It should be noted that Olig2 is manifested at various stages of the development of oligodendrocytes: OPCs, pre-oligodendrocytes or late OPCs, immature pre-myelinating and mature myelinating oligodendrocytes.

In our studies, it was found that a significant loss of Olig2+ oligodendrocytes compared with control cultures (without cuprizone) was observed only with the application of cuprizone on the $18^{\text {th }}$ day of cultivation of the dissociated cerebellar cells culture: from $420.0 \pm 78.4$ to $62.5 \pm 18.2$ cells $(p<0.05)$. The application of the demyelinating agent in these terms of cultivation led to a decrease in the total number of nucleated cells: from $2381.1 \pm 167.7$ to $663.0 \pm 208.4$ cells $(p<0.05)$ (Fig. 9, 11).

It should be noted that more and more researchers consider diffused sclerosis a neurodegenerative disease. Damage to neurons can contribute to the violation of the structure and functioning of the processes of these cells and the formation of such neurological symptoms that are characteristic of multiple sclerosis of humans, such as memory loss, emotions, intelligence and vegetative disorders, etc. [3,30]. The decrease in the content of the total amount of nucleated cells can be explained by the loss of neurons under the impact of cuprizone.

While cuprizone application on the $7^{\text {th }}$ day, significant changes in the number of $\mathrm{Hoechst}^{+}$and Olig2 ${ }^{+}$cells were not detected compared with control cultures: $2381.1 \pm 167.7$ and $2205.0 \pm 392.0 ; 420.0 \pm 78.4$ and $493.0 \pm 41.0$ cells, respectively (Fig. 9, 10). Thus, preliminary data from our studies and literature data confirming that cuprizone does not lead to a decrease in the number of oligodendrocytes that are in its early stages of development $[24,25]$ have been confirmed.

Currently, treatment for multiple sclerosis is aimed at suppressing the immune response and is only moderately effective in reducing the recurrence rate, but does not retard the disease progression, and does not affect remyelination in the central nervous system. Therefore, the search for optimal experimental models for the study of demyelination and remyelination is a topical issue and is important for the understanding of the pathogenesis of multiple sclerosis and the search for new therapeutic approaches to the treatment of this disease.
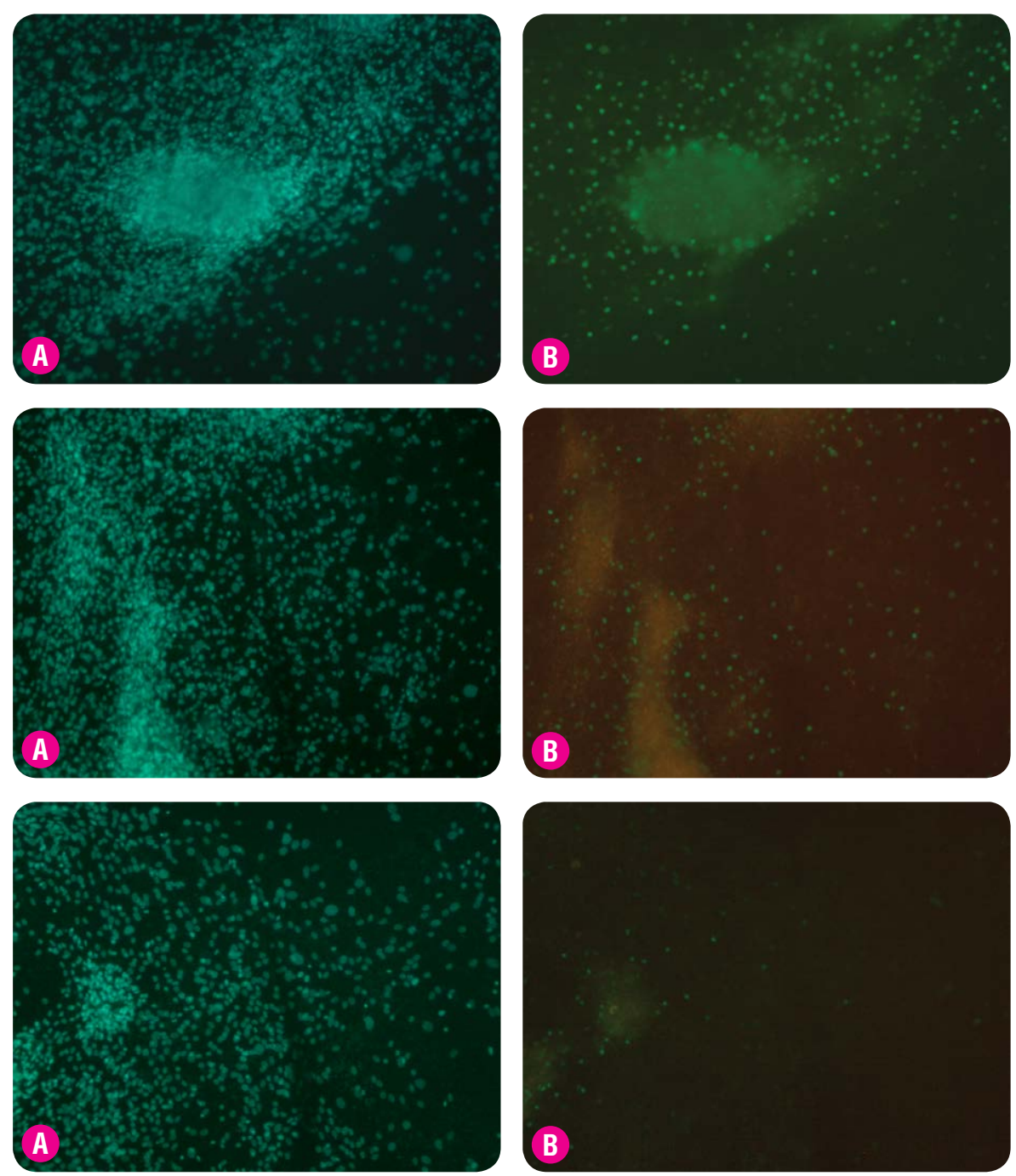
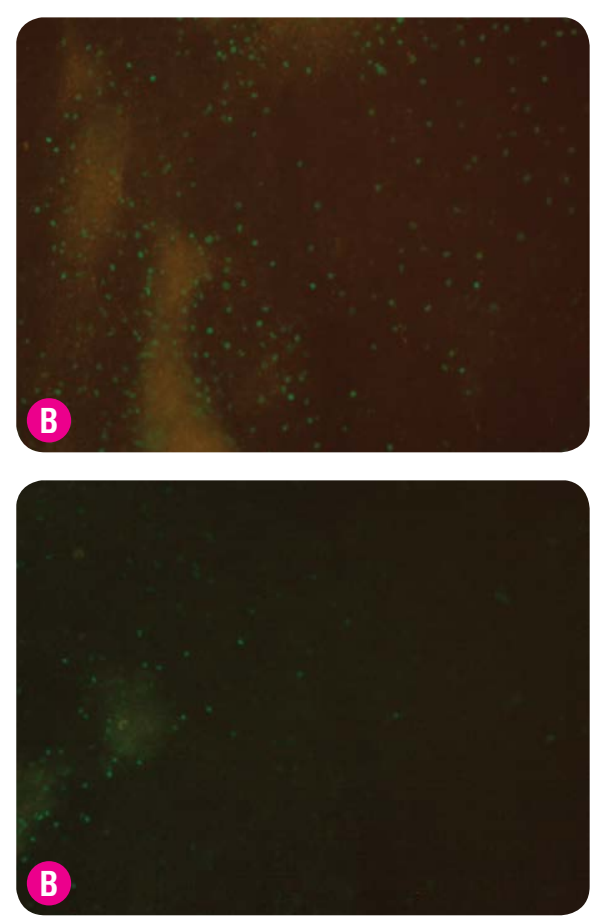

Fig. 9. Microphotographs of dissociated culture of murine cerebellar cells, the $28^{\text {th }}$ day of cultivation; fluorescent microscopy. Immunocytochemical staining for Hoechst 33342 (A, turquoise) and Olig2 (B, green).
Fig. 10. Microphotographs of dissociated culture of murine cerebellar cells under cuprizone application at a dose of $25 \mu \mathrm{M}$ on the $7^{\text {th }}$ day, the $28^{\text {th }}$ day of cultivation; fluorescent microscopy. Immunocytochemical staining for Hoechst 33342 (A, turquoise) and Olig2 (B, green).
Fig. 11. Microphotographs of dissociated culture of murine cerebellar cells under cuprizone application at a dose of $25 \mu \mathrm{M}$ on the $18^{\text {th }}$ day, the $28^{\text {th }}$ day of cultivation; fluorescent microscopy. Immunocytochemical staining for Hoechst 33342 (A, turquoise) and Olig2 ( $B$, green). 


\section{CONCLUSION}

1. In implementation of the toxic cuprizone model of demyelination, the seeding density of cell suspension of murine cerebellum was determined, the conditions of dissociated cerebellar cells cultivation was selected, the survival of the cells in vitro has been achieved until 26-28 days of cultivation.

2. The presence of myelination/demyelination of axons in vitro was confirmed using murine dissociated cerebellar cell culture.

3. Dose-dependent demyelinating effect on axons under application of cuprizone on the $7^{\text {th }}$ day of cultivation of murine dissociated cerebellar cells was detected.

4. It has been shown that cuprizone has inhibitory effect on oligodendrocytes on the $18^{\text {th }}$ day of cultivation.

\section{REFERENCES}

1. Melnyk NO. Struktura dejakih organiv nervovoï ta imunnoï sistem za umov demiєlinizaciï ta remiєlinizaciï [Structure of some organs of the nervous and immune systems in the conditions demyelination and remyelinisation: dissert. doctor of medical sciences]. Kyiv, 2005. 358 p. [In Ukrainian]

2. Pivneva TA. Mekhanizmy demielinizatsii pri rasseyannom skleroze [Mechanisms of demyelination in multiple sclerosis]. Neyrofiziologiya - Neurophysiology. 2009; 41(5):429-437. [In Russian]

3. Suslina ZA, Zavalishin IA. Rasseyannyy skleroz: ot predstavleniy o patogeneze k lecheniyu [Multiple sclerosis: from the concept of pathogenesis to treatment]. Nevrologicheskiy vesnik - Bulletin of Neurology. 2010; 1:6-8. [In Russian]

4. Mishchenko TS, Shulga OD, Bobrik NV, et al. Rozsiyaniy skleroz: global'ni perspektivi [Multiple Sclerosis: Global Perspectives]. Ukr med chasopis. - Ukrainian medical journal. 2014; 101(3):84-87. [In Ukrainian]

5. Miron VE, Kuhlmann T, Antel JP. Cells of the oligodendroglial lineage, myelination, and remyelination. Biochimica et Biophysica Acta. 2011; 1812:184-193.

6. Neyrogennaya differentsirovka stvolovykh kletok [Neurogen differentiation of stem cells]. Edited by Zozulya YuA, Lisyaniy NI. Kiev, 2005. 368 p. [In Russian]

7. Acs P, Kalman B. Pathogenesis of multiple sclerosis: what can we learn from the cuprizone model. Methods Mol Biol. 2012; 900:403-431.

8. Kipp M, Clarner T, Dang J. The cuprizone animal model: new insights into an old story. Acta neuropathol. 2009; 118:723-736.

9. Torkildsen 0, Brunborg LA, Myhr KM, et al. The cuprizone model for demyelination. Acta Neurol Scand Suppl. 2008; 188:72-76.

10. Sachs HH, Bercury KK, Popescu DC, et al. A New Model of Cuprizone-Mediated Demyelination/Remyelination. ASN Neuro. 2014; 6(5). D0I: 10.1177/1759091414551955.

11. Herder V, Hansmann F, Stangel M, et al. Lack of cuprizone-induced demyelination in the murine spinal cord despite oligodendroglial alterations substantiates the concept of site-specific susceptibilities of the central nervous system. Neuropathology and Applied Neurobiology. 2011; 37:676-684.

12. Abakumova TO, Kuz'kina AA, Zharova ME, et al. Cuprizone Model as a Tool for Preclinical Studies of the Efficacy of Multiple Sclerosis Diagnosis and Therapy. Bull Exp Biol Med. 2015; 159(1):111-5. DOI: 10.1007/s10517-015-2903-z.

13. Praet J, Guglielmetti C, Berneman Z, et al. Cellular and molecular neuropathology of the cuprizone mouse model: Clinical relevance for multiple sclerosis. Neurosci Biobehav Rev. 2014; 47:485-505

14. Kipp M, Clarner T, Dang J, et al. The cuprizone animal model: new insights into an old story. Acta Neuropathol. 2009; 118(6):723-736.

15. Basogly H, Boylu NT, Kose H. Cuprizone-induced demyelination in Wistar rats; electrophysiological and histological assessment. Eur Rev Med Pharmacol Sci. 2013; 17(20):2711-2717

16. Barateiro A, Fernandes A. Temporal oligodendrocyte lineage progression: In vitro models of proliferation, differentiation and myelination. Biochimica et Biophysica Acta. 2014; 1843:1917-1929.

17. Pivneva TA, Kolotushkona EV, Mel'nik NA. Mekhanizmy protsessa demielinizatsii i ego modelirovanie [Mechanisms of the demyelination process and its modeling] Neyrofiziologiya - Neurophysiology. 1999; 31(6):497-509. [In Russian]

18. Goldman SA, Kuypers NJ. How to make an oligodendrocyte. Development. 2015; 142:3983-3995.

19. LeBaron FN, Sanyal S, Jungalwala FB. Turnover rate of molecular species of sphingomyelin in rat brain. Neurochem Res. 1981; 6(10):1081-1089

20. Bradl M, Lassmann H. Oligodendrocytes: biology and pathology. Acta Neuropathol. 2010; 119:37-53.

21. Mori S, Leblond CP. Electron microscopic identification of three classes of oligodendrocytes and a preliminary study of their proliferative activity in the corpus callosum of young rats. J Comp Neurol. 1970; 139(1):1-28.

22. Butt $A M$, Ibrahim M, Ruge FM, et al. Biochemical subtypes of oligodendrocyte in the anterior medullary velum of the rat as revealed by the monoclonal antibody rip. Glia. 1995; 14(3):185-197.

23. Baumann N, Pham-Dihn D. Biology of oligodendrocyte and myelin in the mammalian central nervous system. Physiol Rev. 2001; 81(2): 871-927.

24. Wendy C. The neurotoxicant, cuprizone, redards the differentiation of oligodendrocytes in vitro. Neurol Sci. 1999; 168:116-120.

25. Benardais K, Kotsiari A, Skuljiec J, et al. Cuprizone [bis(cyclohexylidenehydrazide)] is selectively toxic for mature oligodendrocytes. Neurotox Res. 2013; 24(2):244250.

26. Osterhout DJ, Wolven A, Wolf RM, et al. Morphological differentiation of oligodendrocytes requires activation of Fyn tyrosine kinase. J Cell Bio. 1999; 145:1209-1218.

27. Lourenço T, Paes de Faria, Bippes CA, et al. Modulation of oligodendrocyte differentiation and maturation by combined biochemical and mechanical cues. 2009. DOI: 10.1038/srep21563.

28. Robinson AP, Rodgers JM, Goings GE, et al. Characterization of oligodendroglial populations in mouse demyelinating disease using flow cytometry: Clues for MS Pathogenesis. Plos One. 2014; $\mathbf{9 ( 9 ) : 1 0 7 6 4 9 . ~}$

29. Patel JR, Klein RS. Mediators of oligodendrocyte differentiation during remyelination. FEBS Letters. 2011; 585:3730-3737.

30. Wegner Cl, Esiri MM, Chance SA, et al. Neurocortical neuronal, synaptic and glial loss in multiple sclerosis. Neurology. 2006; 67(6):960-967.

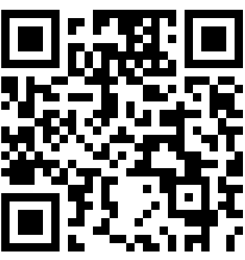

